\title{
Manifestaciones clínicas, factores de riesgo y resultados maternos y perinatales de COVID-19 en el embarazo
}

\author{
Clinical manifestations, risk factors, and maternal and perinatal outcomes of coronavirus disease 2019 in pregnancy
}

\section{Comentado de:}

Allotey J, et al. BMJ. 2020;370:m3320. PMID: 32873575 ${ }^{1}$

\section{Objetivo}

Determinar manifestaciones clínicas, factores de riesgo y resultados maternos y perinatales en mujeres embarazadas con enfermedad por coronavirus 2019 (COVID-19) sospechada o confirmada.

\section{Diseño \\ Revisión sistemática viva y meta-análisis.}

\section{Fuentes de datos}

Medline, Embase, base de datos Cochrane, base de datos COVID-19 de la Organización Muncial de la Salud, Infraestructura Nacional de Conocimiento de China (CNKI) y bases de datos Wanfang desde el 1 de diciembre de 2019 hasta el 26 de junio de 2020, junto con servidores de prepublicación, redes sociales y listas de referencias.

\section{Selección de estudios}

Estudios de cohortes que informan las tasas, las manifestaciones clínicas (síntomas, hallazgos de laboratorio y radiológicos), factores de riesgo y resultados maternos y perinatales en mujeres embarazadas con cCOVID-19 sospechada o confirmada.

\section{Extracción de datos}

Al menos dos investigadores extrajeron los datos de forma independiente y evaluaron la calidad de los estudios. Se realizó un meta-análisis de efectos aleatorios, con estimaciones agrupadas como odds ratios (OR) y proporciones con intervalos de confianza del $95 \%$ (IC 95\%). Todos los análisis se actualizarán periódicamente.

\section{Resultados}

Se incluyeron 77 estudios. En general, el 10\% (IC 95\% $7 \%$ a $14 \%$; 28 estudios, 11.432 mujeres) de las mujeres embarazadas y recién embarazadas que asistieron o ingresaron al hospital por cualquier motivo fueron diagnosticadas con COVID-19 (sospechado o confirmado). Las manifestaciones clínicas más comunes de COVID-19 en el embarazo fueron fiebre (40\%) y tos (39\%). En comparación con las mujeres no embarazadas en edad reproductiva, las mujeres embarazadas y recientemente embarazadas con COVID-19 tenían menos probabilidades de informar síntomas de fiebre (OR 0,43; IC $95 \% 0,22$ a 0,$85 ;$ I $^{2}=74 \%$; 5 estudios; 80.521 mujeres) y mialgias (OR 0,48; IC $95 \% 0,45$ a 0,51 ; $\mathrm{I}^{2}=0 \%$; 3 estudios; 80.409 mujeres) y tenían más probabili- dades de necesitar ingreso en una unidad de cuidados intensivos (UCI) (OR 1,62; IC 95\% 1,33 a 1,96; I ${ }^{2}=0 \%$ ) o ventilación mecánica invasiva (VMI) (OR 1,88; IC $95 \%$ 1,36 a 2,60; I ${ }^{2}=0 \%$; 4 estudios, 91.606 mujeres). Se documentaron 73 muertes, por cualquier causa, de mujeres embarazadas con COVID-19 confirmado $(0,1 \%, 26$ estudios, 11.580 mujeres). Los factores que se asociaron con COVID-19 grave en el embarazo fueron: la mayor edad materna (OR 1,78; IC $95 \%$ 1,25 a 2,55; I ${ }^{2}=9 \%$; 4 estudios; 1.058 mujeres), el índice de masa corporal alto (OR 2,38, IC $95 \%$ 1,67 a 3,39; I $^{2}=0 \%$; 3 estudios; 877 mujeres), la hipertensión arterial crónica (OR 2,0, IC $95 \% 1,14$ a 3,$48 ; I^{2}=0 \% ; 2$ estudios; 858 mujeres) y la diabetes preexistente (OR 2,51, IC $95 \%$ 1,31 a 4,$80 ;$ I $^{2}=12 \%$; 2 estudios; 858 mujeres). La comorbilidad materna preexistente fue un factor de riesgo para el ingreso en UCI (OR 4,21, IC $95 \%$ 1,06 a 16,72; I ${ }^{2}=0 \%$; 2 estudios; 320 mujeres) y VMI (OR 4,48; IC $95 \% 1,40$ a 14,37 ; I ${ }^{2}=0 \%$; 2 estudios; 313 mujeres). La tasa de partos prematuros espontáneos fue de $6 \%$ (IC $95 \% 3 \%$ a $9 \%$; । $2=55 \%$; 10 estudios; 870 mujeres) en mujeres con COVID-19. Las probabilidades de un parto prematuro fueron altas en las mujeres embarazadas con COVID-19 en comparación con las que no tenían la enfermedad (OR 3,01; IC 95\%: 1,16 a 7,85; I ${ }^{2}=1 \%$; 2 estudios; 339 mujeres). Una cuarta parte de todos los recién nacidos de madres con COVID-19 fueron admitidos en la unidad de cuidados intensivos neonatal (25\%); su riesgo de ingreso fue mayor que los nacidos de madres sin COVID-19 (OR 3,13; IC95\%: 2,05 a 4,78; I $^{2}=$ no estimable; 1 estudio , 1.121 recién nacidos).

\section{Conclusión}

Las mujeres embarazadas tienen menos probabilidades de manifestar síntomas de fiebre y mialgia relacionados con COVID19 que las mujeres no embarazadas en edad reproductiva, y son potencialmente más propensas a necesitar tratamiento en UCI para esta entidad. Las comorbilidades preexistentes, la edad materna alta y el índice de masa corporal alto parecen ser factores de riesgo de enfermedad grave. Las tasas de nacimientos prematuros son más altas en las mujeres embarazadas con COVID-19 en comparación con las mujeres embarazadas que no la padecen.

Fuente de financiamiento/Conflicto de interés de los autores: Parcialmente financiado por la Organización Mundial de la Salud. Los autores declararon no tener conflictos de interés. Registro de la revisión sistemática en PROSPERO CRD42020178076.

\section{Comentario}

Las publicaciones sobre COVID-19 en el embarazo han aumentado considerablemente a través de informes de casos individuales, series de casos, estudios observacionales y revisiones sistemáticas. Al 26 de junio de 2020 , se han publicado más de 86 revisiones en esta área y otras 94 fueron registradas en PROSPERO ${ }^{1}$

De los 77 estudios incluidos en esta revisión, 26 (34\%) eran de los EE.UU., 24 de China (31\%) y sólo dos de Latinoamérica (Brasil y México). El $67 \%$ (37/55) de los estudios de cohortes comparativos evaluados mediante la escala de Newcastle Ottawa tenían un bajo riesgo de sesgo global. Todos los hallazgos comparativos se basaron en un pequeño número de estudios, a pesar de los grandes tamaños de muestra, y presentaban una heterogeneidad sustancial. 
Mientras que las pruebas de SARS-CoV-2 en mujeres no embarazadas se basan en la presencia de síntomas o antecedentes de contacto, en mujeres embarazadas generalmente se realizan cuando están en el hospital por razones que pueden o no estar relacionadas con el COVID-19, y parecen tener un mayor riesgo de requerir ingreso en una UCI o VMI. Las tasas de muerte fetal y neonatal son bajas en mujeres con COVID-19 presunto o confirmado. Las políticas locales sobre observación y cuarentena de los bebés expuestos al SARS-CoV-2 podrían haber influido en estas tasas.

Esta revisión sistemática viva, actualizada continuamente a intervalos regulares, parece el abordaje más apropiado durante la actual pandemia, momento en que la evidencia se produce y publica vertiginosamente. Entre las fortalezas de este estudio se destaca la exhaustiva y continua búsqueda de evidencia y los múltiples análisis comparativos que permitieron evaluar de manera integral la asociación entre el embarazo y los resultados relacionados con esta nueva enfermedad. También presenta limitaciones. Los estudios primarios utilizaron muestreos variados para identificar a las mujeres con COVID-19 tanto sospechada como confirmada, e informaron principalmente sobre mujeres embarazadas que requirieron visitas al hospital, incluso para el parto, lo que afecta la generalización de las estimaciones. Esto podría haber sobrestimado la verdadera prevalencia de la enfermedad en mujeres embarazadas. Además, las definiciones de síntomas, pruebas y resultados fueron heterogéneas. Por otro lado, pocos estudios informaron resultados por trimestre de gestación, y se reportaron deficientemente los criterios de cesárea, ingresos a la unidad neonatal y las causas del parto prematuro, haciendo difícil separar el efecto iatrogénico del verdadero impacto de la enfermedad.

Al igual que en la población general, el índice de masa corporal alto y la comorbilidad preexistente parecían ser factores de riesgo de gravedad ${ }^{2}$.

\section{Conclusiones del comentador}

Las mujeres embarazadas con COVID-19 pueden requerir más frecuentemente ingreso a UCl y VMI en comparación con las no embarazadas. Las gestantes con comorbilidades preexistentes, obesidad y mayor edad deberían ser consideradas como un grupo de alto riesgo para esta nueva enfermedad, requiriendo una atención prenatal multidisciplinaria. Se necesitan más estudios para evaluar de manera robusta las complicaciones maternas y neonatales así como la asociación con otros factores de riesgo, al igual que la etnia y los factores de riesgo específicos del embarazo, como la preeclampsia y la diabetes gestacional. También se requieren más datos por trimestre de exposición, incluido el período periconcepcional, para determinar los efectos del COVID-19 en los resultados tempranos del embarazo, el crecimiento fetal y el riesgo de muerte fetal.

Los meta-análisis con datos de participantes individuales de las cohortes serían fundamentales para evaluar tanto la presentación diferencial como los resultados por factores de riesgo subyacentes y determinar los efectos diferenciales de las intervenciones para reducir las tasas de complicaciones.

Agustín Ciapponi [ Servicio de Medicina Familiar y Comunitaria, Hospital Italiano de Buenos Aires; Centro Cochrane Argentina, Instituto de Efectividad Clínica y Sanitaria. aciapponi@gmail.com ]

Ciapponi A Manifestaciones clínicas, factores de riesgo y resultados maternos y perinatales de COVID-19 en el embarazo. Evid Actual Pract Ambul. 2020;23(4):e002094. Comentado de: Allotey J, et al. Clinical manifestations, risk factors, and maternal and perinatal outcomes of coronavirus disease 2019 in pregnancy: living systematic review and meta-analysis. BMJ. 2020;370:m3320. PMID: 32873575

\section{Referencias}

1. Allotey J, Stallings E, Bonet M, et al. Clinical manifestations, risk factors, and maternal and perinatal outcomes of coronavirus disease 2019 in pregnancy: living systematic review and meta-analysis. BMJ. 2020;370:m3320. Available from: 10.1136/bmj.m3320.

2. Docherty AB, Harrison EM, Green CA, et al. Features of $20133 \mathrm{UK}$ patients in hospital with covid-19 using the ISARIC WHO Clinical Characterisation Protocol: prospective observational cohort study. BMJ. 2020;369:m1985. Available from: 10.1136/bmj.m1985. 